\title{
Implicancias de las técnicas de medición de la actividad cerebral en la cognición: ¿El tiempo o el espacio?
}

\section{Implications of mediation techniques of cerebral activity in cognition: ¿Time or Space?}

Pedro Díaz

\begin{abstract}
Resumen
Se propone una discusión respecto a las interpretaciones teóricas que se hacen en las ciencias cognitivas, a partir de los hallazgos permitidos por las nuevas tecnologías para medir y observar la actividad cerebral. Se exponen los promisorios avances permitidos por las últimas técnicas de neuroimagen o "Técnicas de Espacio" como el TEP, el fMRI y otros, para delimitar los circuitos y las zonas donde se concentran variadas actividades cognitivas altas. Se contrapone esto con las características de las "Técnicas de Tiempo", como el electroencefalograma (EEG) o el magneto encefalograma (MEG), y algunos avances empíricos que estas han permitido, alimentando teorías predominantemente asociadas a los sistemas dinámicos. Finalmente, se comentan las implicancias de ambos tipos de técnicas en las teorías de la cognición, llamando la atención sobre la necesidad de analizar qué estamos viendo con cada técnica, y cómo lo que estamos viendo afecta nuestras concepciones teóricas.

Palabras clave: fMRI, EEG, neurociencia cognitiva.
\end{abstract}

\begin{abstract}
Considering the discoveries allowed by new technologies to observe and measure neural activity, a discussion is proposed about theoretical interpretations in cognitive sciences. The author comments these interpretations in the frame of the promissory advances in way to delimitate circuits and zones where high cognition activities are concentrated, advances that are predominantly possible with
\end{abstract}

Magíster en Estudios Cognitivos (t), Centro de Estudios Cognitivos CEC, Facultad de Humanidades, Universidad de Chile.p.diaz.cartes@gmail.com 
the development of "Space Techniques", like the MRI, the fMRI and others. This is opposed with the "Time Techniques", (like electroencephalograph (EEG)) empirical approaches and discoveries, that have fed cognitive theories associated with Dynamic Systems. Finally, influences of both types of techniques in cognition are commented, paying special attention to analyze what we observe with each technique, and how that influences our theoretical conceptions.

Key words: fMRI, EEG, cognitive neuroscience.

\section{Tiempo o espacio: inicios}

Los avances tecnológicos de la neurología han sido considerables durante los últimos años y han puesto a disposición de clínicos y científicos un gran set de herramientas para detectar actividad cerebral como correlato de conductas y actividades cognitivas "altas". Este desarrollo ha impulsado un "boom" de publicaciones que utilizan estas nuevas tecnologías, y los hallazgos son sorprendentes (Gazzaniga et al., 2002). Sin embargo, se hace necesario reflexionar sobre lo que estamos midiendo y sus implicancias, pues estas técnicas tienen características diferenciales que limitan el campo de especulación, y que muchos optimistas olvidan.

La búsqueda de evidencia en torno a esta resbalosa relación entre mente y cerebro no es nueva; desde antiguo tanto filósofos como psicólogos y biólogos han intentado dar cuenta de ella. Posner y Raichle (1999) comentan que ya en el año 1863 había científicos que planteaban directamente que estructuras individuales y simples en el cerebro eran las culpables de conductas específicas (como es el caso de un reflejo), mientras que otros colegas posteriores afirmaban que lo anterior era artificial, pues las partes del sistema nervioso están conectadas entre sí y ninguna zona es capaz de reaccionar sin afectar y ser estimulada por otras zonas. Como podemos observar, desde los inicios de la Ciencia Cognitiva han existido dos visiones contrapuestas, que fueron evolucionando a lo que actualmente se conoce como teorías localizacionistas (aquellas teorías que buscan aislar 
estructuras en el cerebro a partir de las funciones específicas que desempeñan, intentando detectar "órganos" mentales muy cercanos a las ideas del cognitivismo computacionalista) y teorías dinámicas (partidarios de analizar el cerebro como un sistema complejo con miríadas de interacciones reciprocas, y de extrapolar a la neurociencia cognitiva la capacidad explicativa de las teorías de sistemas dinámicos en la termodinámica o en otras disciplinas) (Van Gelder, 1998; Kelso, 2003).

Paralelo a lo anterior, la tecnología también evolucionaba. Hans Berger, en 1929, es de los primeros científicos que logra un registro en EEG, midiendo la actividad eléctrica postsináptica, (técnica que ha seguido desarrollándose hasta nuestros días). En los años 70, por otro lado, se potencian tecnologías de rayos $\mathrm{X}$, obteniéndose las primeras imágenes con la Tomografía Computarizada (TC). Lo anterior ha dado paso a las actuales Imágenes por Resonancia Magnética (MRI y fMRI), que utilizan las capacidades magnéticas de los átomos para obtener imágenes de gran resolución, ya sea de estructuras estáticas (MRI) o de la actividad de ciertas áreas del encéfalo, midiendo el aumento o disminución del flujo sanguíneo de estas a través de las propiedades magnéticas de la hemoglobina (fMRI) (Gazzaniga et al., 2002; Posner y Raichle, 1999)

\section{Técnicas de Tiempo y Técnicas de Espacio}

Tomando en cuenta lo anterior, y para efectos del análisis, se ha decidido dividir la diversidad de técnicas desarrolladas en la actualidad, usando como punto de referencia su fortaleza en cuanto a resolución, ya sea temporal o espacial. Por esto entonces, hablaremos de Técnicas de Espacio y Técnicas de Tiempo.

La mayor resolución espacial de las Técnicas de Espacio, permite obtener imágenes con lujo de detalles y gran precisión, delimitando incluso zonas específicas que se activan ante una tarea cognitiva. Dentro de este tipo de técnicas, está la Tomografía por Emisión de Positrones (TEP), Imágenes por Resonancia Magnética (Magnetical Resonance Imaging, o MRI, y Functional Magnetical Resonance Imaging, o fMRI), técnicas de estudio 
neurológico por lesiones cerebrales, estudio de actividad eléctrica de neuronas individuales o de pequeños grupos de neuronas, y Estimulación Magnética Transcraneana (EMT). Paralelamente, las Técnicas de Tiempo, que poseen mejor resolución temporal y permiten medir la actividad cognitiva en tiempo real (Gazzaniga et al., 2002), están representadas por el Electroencefalograma (EEG) y el Magnetoencefalograma (MEG). A continuación se analizan en detalle las características de ambas clasificaciones.

\section{a) Resolución espacial:}

De la Técnica de Espacio más potente e innovadora, el fMRI, se puede decir que no mide directamente la actividad neuronal, sino que solamente los cambios metabólicos celulares que ocurren con ésta, bajo el simple principio de que aquella zona que está más activada, requerirá una mayor cantidad de oxígeno. Es por esto que se marca la hemoglobina con un isótopo radiactivo, haciendo visible aquellas zonas donde la irrigación sanguínea sufre una variación estadísticamente significativa. El fMRI posee una resolución espacial de $3 \mathrm{~mm}^{3}$, mientras sus predecesoras, como el TEP, tienen una resolución espacial de aprox. 5 a $10 \mathrm{~mm}^{3}$, junto a un sin fin de desventajas respecto al fMRI, pues somete a los sujetos a altas concentraciones de radiación y no se pueden hacer varias mediciones en períodos cortos de tiempo (Gazzaniga et al., 2002). Las lesiones cerebrales - otra técnica de espacio - dan excelente resolución espacial, pero su complicación es que son muy invasivas y en humanos deben hacerse con el agente cognitivo ya muerto. La técnica con mejor resolución espacial es el registro de la actividad eléctrica de neuronas individuales o de grupos pequeños, pero su problema es que al analizar grupos tan pequeños se pierde la capacidad de dar cuenta de patrones más masivos. Otra técnica es la EMT; crea lesiones cerebrales "virtuales" que son reversibles, y cuya resolución es de 1 a $1,5 \mathrm{~cm}^{2}$ (puede producir estas lesiones o inactivaciones a nivel de corteza en este radio).

En el caso de las Técnicas de Tiempo la resolución espacial no es mejor. En el EEG, es de alrededor de los $3 \mathrm{~mm}$, y mide la actividad eléctrica de grupos de neuronas y no de neuronas específicas. Sin embargo, hay 
que tomar en cuenta que no toda la actividad eléctrica o magnética que se registra es propia del cerebro; es necesario limpiar la señal de muchas otras señales (de los propios electrodos, de la instalación eléctrica del lugar donde se mide, de movimientos musculares, especialmente oculares, etc) y en el caso del EEG, solo es posible medir la actividad de las zonas externas de la corteza; aquella actividad que se encuentra al interior de los variados surcos y recovecos propios del cerebro, es solo estimable. Algo semejante pasa con el Magneto encéfalograma (MEG) (Gazzaniga et al., 2002).

\section{b) Resolución temporal:}

La resolución temporal no es tan deslumbrante como la espacial en el caso de las Técnicas de Espacio. Debido al mismo proceso que se necesita llevar a cabo en cada técnica - que incluye por ejemplo la inyección de isótopos radiactivos, o el procesamiento de la información entrante en un software, necesario para obtener una imagen relativamente nítida, lo cual indefectiblemente implica un "delay" temporal importante para el estudio de la cognición - las Técnicas de Espacio tienen una resolución temporal que parte desde un segundo en el fMRI a horas en el TEP. El caso de las lesiones cerebrales es, obviamente peor, pero no así el de la EMT, que tiene una resolución temporal de 1 segundo hacia arriba.

En cambio, las Técnicas de Tiempo tienen excelente resolución y pueden medir la actividad eléctrica neuronal en milisegundos. Esto permite estudiar los "potenciales eléctricos relacionados con eventos" muy específicos (PRE), casi en el momento preciso en que se producen. Ejemplos son el PRE denominados P300 (potencial característico asociado básicamente a estímulos auditivos discontinuos) y el N400 (asociado a estímulos lingüísticos con errores semánticos o sintácticos, e incluso a estímulos gestuales incoherentes) (Reid y Striano, 2008; Friedrich y Friederici, 2004), además de la sincronía entre potenciales en las mismas o distintas zonas del cerebro (Slotnick, et al., 2002; Spencer, et al., 2004) en una ventana que puede ir desde 0 milisegundos hasta 130 (donde aparecen los potenciales más tempranos) (Gazzaniga et al., 2002). 


\section{c) Medición en tiempo real}

Como vemos, la resolución temporal de las Técnicas de Espacio no es suficiente al compararla con la resolución de las Técnicas de Tiempo, y menos en comparación con la velocidad de los procesos cognitivos, los cuales ocurren en unas ventanas temporales de milisegundos (Gazzaniga et al., 2002). Es por esto que las Técnicas de Espacio están en desventaja con las de tiempo en su capacidad de dar cuenta de actividad cerebral en tiempo real pues, a pesar de su precisión, no pueden proporcionar información on line, es decir, registrar lo que ocurre en la corteza en el momento mismo en el que se está produciendo el fenómeno cognitivo.

\section{d) Imágenes dinámicas}

Por imágenes dinámicas, se entiende la capacidad de la técnica de mostrar la evolución de la activación cerebral en el tiempo, en una especie de "video" que permita observar los cambios que se van produciendo en relación con las actividades cognitivas, con el máximo de resolución temporal y espacial. Al parecer, esta capacidad no ha sido una meta primordial de ninguna de las técnicas planteadas y ha sido una herramienta que pocos han utilizado en sus estudios (un ejemplo es el de Lutz, Lachaux, Martinerie, Varela, 2002), siendo un aspecto interesante que permitiría observar patrones de activación de toda la corteza, que sean característicos de ciertos estímulos intrínsecos y extrínsecos.

Respecto a esto, las observaciones de cada tipo de técnicas son limitadas por sus resoluciones. En el caso de las Técnicas de Tiempo, es posible unificar las mediciones de los electrodos a través de los segundos, de manera de hacer un mapeo dinámico relativamente on line para observar cómo evolucionan las señales eléctricas a través de la corteza y analizar patrones, por ejemplo, de sincronía o desincronía neuronal. En el caso de las Técnicas de Espacio, esto es difícil, pues sólo se obtienen imágenes estáticas con un desfase de tiempo importante que no permite apreciar la evolución temporal de fenómenos corticales. 


\section{Los promisorios avances.}

La cantidad de investigaciones con las Técnicas de Espacio son innumerables. Por ejemplo, en el caso de la imagenología son sabidos los avances en delimitar las áreas implicadas en trabajo visual, reconocimiento de rostros, de colores, de formas, etc. También se conoce el éxito en delimitar las áreas implicadas en el control motor, sensorial y las zonas implicadas en el reconocimiento de sonidos relacionados con el lenguaje (Gazzaniga et al., 2002).

El caso de las Técnicas de Tiempo, además de los ya mencionados PRE, se han alcanzado grandes avances en describir patrones de sincronía (Engel, Fries y Singer, 2001; Penny, Kiebel, Kilner y Rugg, 2002) y han sido útiles para registrar correlatos neurales de la conciencia y patrones anormales de oscilación, percepción y cognición en la esquizofrenia (Van der Stelt, Belger y Lieberman, 2004; Spencer et al., 2004), además de eventos relacionados con el sueño y su rol en la cognición (Winson, 2002; Hobson y Pace-Schott, 2002), y la detección de patrones patológicos de onda, característicos de la epilepsia.

Lo anterior, sin embargo, se da en un marco en que la cantidad de investigaciones que prefieren la imagenología como técnica de medición de la actividad cerebral podría ser mucho mayor. Fellows y colaboradores (2005), reportan que, observando la evolución de la cantidad de artículos publicados que utilizan métodos de lesión, comparados con métodos de imagenología (en los años 1993, 1997 y 2001), se ve un aumento considerable de estos últimos con el paso de los años. Además, los artículos que utilizaron imagenología se citan considerablemente más (el triple $)^{2}$ y aparecen en aquellos journals de mayor impacto. A pesar de que esta comparación es entre el método de lesión y la imagenología (los cuales incluyo en el presente ensayo bajo la clasificación de Técnicas de Espacio), no se han

En el estudio se tomaron artículos que utilizan imagenología o lesión y que aparecieron en los años 1993 y 1997. A partir de estos, se analizó las veces que fueron citados en otros artículos cada año desde la fecha de publicación hasta septiembre de 2003. Para más detalles, en el articulo de Fellows et al. se muestran gráficos elaborados con los promedios de citaciones por ańo, y la diferencia es evidente. 
encontrado revisiones que los comparen con las de tiempo, pero se sospecha que la diferencia es importante.

\section{Sistemas dinámicos vs. enfoque modular}

Por otro lado, resulta llamativo notar tendencias muy marcadas en los marcos teóricos y las conclusiones que se extraen de estos estudios, dependiendo del tipo de técnica (de tiempo o espacio) que se use. Por ejemplo, está el caso del enfoque de los sistemas dinámicos. El uso de este enfoque en el área no es algo nuevo, y al parecer genera muchas expectativas entre los teóricos de la cognición alternativa (Kelso, 2003; Tschacher, Dauwalder y Haken, 2003). Algunos de los planteamientos de este enfoque en la cognición son las siguientes: a) Los sistemas cognitivos pueden ser vistos como sistemas dinámicos, gobernados por ecuaciones diferenciales; b) En cognición, los científicos deben tomar en cuenta cómo cambia el sistema, y establecer los estados que toma el sistema en este cambio; c) La cognición puede ser entendida de forma geométrica, en torno a la posición de un estado cognitivo respecto de otro estado o respecto a cualquier rasgo del sistema. Lo anterior, por lo tanto, postula más que una arquitectura cognitiva, un "paisaje", compuesto de estados y atractores; d) La ciencia cognitiva debe describir la estructura que manifiesta un sistema mientras sus procesos evolucionan temporalmente; e) El procesamiento mental es predominantemente en paralelo, al contrario del procesamiento serial, que es defendido por la visión computacionalista de la cognición; f) Inputs - outputs vs. continuidad del procesamiento: que implica que el procesamiento cognitivo ocurre en un continuo fluir, que no comienza (input) ni termina (output) en ningún momento; g) Estados vs. coupling: que implica que los sistemas cognitivos funcionan en directa relación y acople con el ambiente y no ante un input específico o ante las reglas internas del sistema; y h) Los dinamicistas identifican la representación mental, centro de la cognición clásica, con atractores y trayectorias, más que con modelos simbólicos formales (Van Gelder, 1998). 
Lo interesante es que los preceptos mencionados tienden a ser usados como marco conceptual en aquellas investigaciones que utilizan las Técnicas de Tiempo. Algunos ejemplos posibles de mencionar son investigaciones sobre la conciencia (Edelman, 2003; Lutz et al., 2002), y observaciones en la sincronización de actividad oscilatoria en el cortex visual en gatos (Konig, Ángel y Singer, 1995). En el primer caso, Edelman propone abarcar las diversas manifestaciones y fenómenos asociados a la conciencia con un enfoque que pueda naturalizarla, basándose en principios evolucionistas. Por lo tanto, en su artículo comenta los hallazgos de la neurociencia respecto a este tema en monos y humanos, que muestran que no sería propiedad de una zona específica, sino que el resultado de la interacción dinámica y paralela entre diversos grupos de neuronas al interior del SNC. Lutz y colaboradores, por su parte, intentan establecer un correlato entre fenómenos corticales medidos en EEG y algunas técnicas de investigación de la subjetividad en primera persona, usadas por la psicología y con un marco fenomenológico, intentando buscar patrones de activación cortical comunes. Una de las conclusiones a la que los investigadores llegaron para investigar esto, es que los métodos de neuroimagen (TE), a pesar de que responden a protocolos estrictos de medición, no sirven para captar este tipo de fenómenos, que ocurren en un contexto de flujo conciente, en el cual diversos factores influyen en paralelo y acoplados con el tiempo. Además, los autores utilizan la capacidad de generar imágenes dinámicas (punto 2.d. del presente texto), a la que denominan DNS y que es más poderosa en las técnicas de tiempo. Lo anterior les permitió apreciar sincronías entre electrodos a nivel de banda gamma situados en el lóbulo frontal.

Finalmente, Konig plantea directamente en la introducción de su trabajo que el fenómeno de sincronización detectado con EEG, apoya la idea de que se establecen relaciones dinámicas entre grupos de neuronas, incluso distantes entre sí. Esto proporciona una solución flexible a los investigadores para las complicaciones que presentan funciones cognitivas como la segmentación de escenas o representación de objetos. Incluso esta sincronización entre zonas distantes es tan íntima (es decir, en coupling, otro término dinámico), que al alterarla con algún dispositivo, se ve afectada incluso la percepción. Vale decir entonces que la percepción, el 
procesamiento de la información cognitiva y la actividad neuronal se dan al mismo tiempo y en paralelo, afectándose entre sí en constante retroalimentación (Roelfsema, Konig, Engel, Sireteanu y Singer, 1994, en Konig et al., 1995). Estos y otros estudios, (entre ellos Engel et al., 2001; Penny et al., 2002; Van der Stelt et al., 2004; Spencer et al., 2004) muestran la influencia de los conceptos propios de estas teorías.

En resumen, el rol que la teoría de los sistemas dinámicos aplicados a la cognición le da al tiempo, (a pesar de que otros autores como Eliasmith (1998) planteen que la atención a esta variable no es exclusiva de los sistemas dinámicos), y la predilección por establecer las formas en que las variables y parámetros del sistema evolucionan espacio - temporalmente, buscando siempre patrones de procesos (Van Gelder, 1998), hace evidente el porqué existiría esta preferencia. Una técnica como el EEG permite apreciar fácilmente los aspectos que destacan los dinamicistas como esenciales en el funcionamiento mental, e incluso es posible mapear las señales obtenidas con los electrodos a través de procedimientos matemáticos y estadísticos, y estructurar topografías en 2D, 3D e incluso 4D (incluyendo la variable tiempo), que hacen posible comparar áreas de la corteza entre sí para hallar trayectorias, atractores y sincronía.

Por otro lado, en el otro extremo, los investigadores que utilizan las Técnicas de Espacio, establecen conclusiones muchas veces cercanas a las teorías clásicas, computacionalistas y representacionalistas de la cognición, buscando zonas específicas que participan en actividades cognitivas delimitadas. Esto apoya la visión modular de la mente, propia del computacionalismo clásico, el cual según Van Gelder, o Clark (1997), es eminentemente homuncular, es decir, ve la mente formada por partes o componentes que cumplen una subtarea rígida, que le es propia y la define. Esto es semejante al funcionamiento de departamentos o comités dentro de sistemas burocrácticos, que interactúan traspasándose información a través de símbolos (Van Gelder, 1998). Lo anterior desemboca en la propuesta de que la mente está constituida por "módulos" con distintas capacidades cognitivas, y que en interacción con otros módulos, procesan símbolos a partir de reglas. Lo que hay en estos módulos puede ser desde memoria, hasta creencias, actitudes e intencionalidad (Newell y Simon, 1979; Fodor, 1988). 
Sabido esto, es fácil entender por qué muchos de los hallazgos y las conclusiones que los investigadores extraen con las Técnicas de Espacio, utilicen el marco conceptual computacionalista. Estas técnicas dan escasa o nula información sobre el tiempo, y menos sobre la evolución de procesos cognitivos, sino que extraen instantáneas de la realidad, mostrándola engañosamente estática. Un pequeño ejemplo son los estudios de Chouinard y Paus (2006) y Wandell, Brewer y Dougherty (2005).

Sin embargo, cabe destacar que esta tendencia se ha roto en el último tiempo, observando fenómenos de plasticidad y mutlimodularidad con técnicas de espacio, en áreas asociadas por ejemplo con la interacción estrecha entre corteza visual y auditiva (Lehman et al., 2006; Renier et al., 2005) y plasticidad en zonas sensoriomotoras primarias (Feldman y Brecht, 2005; Wuhle, Fahlbusch y Braun, 2006), las cuales no cumplirían solo una función específica sino que participarían en varias tareas, y sufrirían cambios dependiendo del tipo de tarea asignada. Incluso Damasio (1994, en Clark, 1997), también con técnicas predominantemente de espacio, establece el concepto de áreas de convergencia neuronal, es decir, atractores (concepto propio de los sistemas dinámicos), o grupos de neuronas en las cuales convergen señales electroquímicas que son redistribuidas gracias a que las neuronas de estas zonas conectan áreas corticales distantes entre sí.

Aún así, persiste la aparente preferencia de los partidarios de las Técnicas de Espacio por buscar zonas corticales o cerebrales unitarias y establecer sus funciones específicas en la actividad sensorial, perceptual y cognitiva (tarea que Fellows et al., 2005 plantea como una de las más grandes búsquedas de la neurociencia) lo cual, caricaturizando un poco, es un ánimo de "frenología sofisticada", claramente influido por las limitaciones de la técnica utilizada.

\section{Discusión}

En definitiva, las observaciones anteriores se explican por un factor poco explorado, y que tiene relación con las características y los límites de las técnicas utilizadas. Resulta obvio, a la luz de lo visto, que aquellos que 
prefieran Técnicas de Tiempo tengan un mayor acceso a plantear explicaciones teóricas que tomen esta característica como la esencial, e ideales para este propósito son las ideas de los sistemas dinámicos. Mientras, los conceptos y hallazgos que surgen con las Técnicas de Espacio y la visión localizacionista, encajan más con la ciencia cognitiva clásica representacionalista y modular, cuyas características ya mencionamos.

El uso de estos marcos teóricos pareciera estar íntimamente relacionado con cada tipo de técnica, y es necesario tomar en cuenta estas observaciones al momento de describir el funcionamiento cognitivo. $\mathrm{Si}$ estas no se toman en cuenta, es posible que estructuremos teorías sobre la cognición que en su base están más en función de factores propios de estas técnicas que de lo verdaderamente "mental". Mientras no tengamos una máquina que posea una resolución espacial y temporal perfecta o al menos que funcione en torno a los ritmos propios y característicos de la actividad cerebral, las conclusiones que hagamos estarán sesgadas, cumpliéndose lo que ya Niels Bohr afirmó en una conferencia en Como, Italia, en 1927: "En la descripción quántica no se puede observar el 'estado del sistema' sin influenciarlo significativamente" (Holton, 1982, p. 119), planteando como ejemplo el microscopio de rayos gamma de Heisenberg, en el que la forma de "observar" el desplazamiento de un electrón, es cuando dispersa rayos gamma. Sin embargo, si hacemos que disperse rayos gamma, inevitablemente el mismo electrón se desvía de su trayectoria original. Por lo tanto, la única forma de que el científico y sus máquinas queden al margen de lo observado en este caso, es cuando no se observa nada en absoluto. En el momento en que el científico tiene sus instrumentos en la mesa de trabajo y se dispone a observar un objeto, concluye Holton, todos estos elementos se transforman en un todo inseparable (Holton, 1982)

En definitiva, los instrumentos en sí delimitan, determinan y muchas veces alteran (como el caso del microscopio de Heisenberg) el objeto observado. 


\section{Bibliografía}

Chouinard, P.; Paus, T. (2006). The Primary Motor and Premotor Areas of the Human Cerebral Cortex. The neuroscientist, 12 (2), 143 - 152.

Clark, A. (1997). Being there, putting brain, body and world together again. EEUU: Bradford books / MIT press.

Edelman, G. (2003). Naturalizing Conciousness: A Theoretical Framework. PNAS, 100 (9), $5520-5524$.

Eliasmith, C. (1998). The Third Contender: A Critical Examination for the Dinamicist Theory of Cognition. En Thagard, P. (Ed.) Mind Readings, Cambridge: MIT press.

Engel, A.; Fries, P.; Singer, W. (2001). Dynamic Predictions: Oscillations and Synchrony in Top Down Processing. Nature Reviews, Neuroscience, 2, $704-716$.

Feldman, D.; Brecht, M. (2005). Map Plasticity in Somatosensory Cortex. Science, 310, $810-815$.

Fellows, L.; Heberlein, A.; Morales, D.; Shivde, G.; Waller, S.; Wu, D. (2005). Method Matters: An Empirical Study of Impact in Cognitive Neuroscience. Journal of Cognitive Neuroscience, 17(6), 850 - 858.

Fodor, J. (1988). Psicosemántica. Cambridge: Bradford books / MIT press.

Friedrich, M.; Friederici, A. (2004). N400 like semantic incongruity effect in 19 - month olds: processing known words in picture contexts. Journal of cognitive neuroscience, 16(8), $1465-1477$.

Gazzaniga, M.; Ivry, R.; Magnun, G. (2002). The Methods in Cognitive Neuroscience. En Cognitive Neuroscience: The Biology of The Mind, (69-120), New York: W.W. Norton \& Company.

Hobson, J.A.; Pace-Schott, E. (2002). The Cognitive Neuroscience of Sleep: Neuronal Systems, Consciousness and Learning. Nature reviews, Neuroscience, 3, $679-693$.

Holton, G. (1982). Ensayos Sobre el Pensamiento Cientifico en la Época de Einstein. México: Editorial Alianza Universidad.

Kelso, J.A. (2003). Cognitive Coordination Dynamics. En Tschacher W.; Dauwalder, J.P. (Eds.) Dynamical systems Approaches to embodied cognition. Singapore: World scientific.

Konig, P.; Ángel, A.; Singer, W. (1995). Relation between Oscillatory Activity and Long Range Synchronization in Cat Visual Cortex. Proc. Nac. Academy, 92, $290-294$.

Lehman, C.; Herdener, M.; Espósito, F.; Huble, D.; Di Salle, F.; Sheffler, K.; Bach, D.; Federspiel, A.; Kretz, R.; Dierks, E.; Seifritz, T. (2006). Differential Patterns Of Multisensory Interactions In Core And Belt Areas Of Human Auditory Cortex. Neuroimagen, 31(1), 294 - 300.

Lutz, A.; Lachaux, J.P.; Martinerie, J.; Varela, F. (2002). Guiding the Study of Brain Dynamics by Using First Person Data: Synchrony Patterns Correlate With Ongoing Conscious States during Simple Visual Task. PNAS, 99(3), $1286-1591$. 
Newell, A.; Simon, H. (1979). Computer Science as Empirical Enquiry: Symbols and Search. Communications of the Association for Computing Machinery, 19(3), $113-126$.

Penny, W.; Kiebel, S.; Kilner, J.; Rugg, M. (2002). Event Related Brain Dynamics. Trends in Neuroscience, 25(8), 387 - 389.

Posner, M.I.; Raichle, M.E. (1999). Images of the Mind. New York: Scientific American Library.

Reid, V.; Striano, T. (2008). N400 involvement in the processing of action secuences. Neuroscience letters, 433, $93-97$.

Renier, L.; Collignon, O.; Poirier, C.; Tranduy, D.; Vanlierde, A.; Bol, A.; Veraart, C.; De Volder, A. (2005). Crossmodal Activation of Visual Cortex during Depth Perception using Auditory Subtitution of Vision. Neuroimage, 26, 573 - 580.

Slotnick, S.; Moo, L.; Kraut, M.; Lesser, R.; Hart, J. (2002). Interactions between thalamic and cortical rythms during semantic memory recall in human. PNAS, 99(9), 6440 - 6443.

Spencer, K.; Nestor, P.; Perlmutter, R.; Niznikievicz, M.; Klump, M.; Frumin, M.; Shentson, M.; Mc Carley, R. (2004). Neural Synchrony Indexes Disordered Perception and Cognition in Schizophrenia. PNAS, 101(49), 17288 - 17293.

Tschacher, W.; Dauwalder J.P.; Haken, H. (2003). Self Organizing Systems Show Apparent Intentionality. En W. Tschacher \& J.P. Dauwalder (eds.) Dynamical Systems Approaches To Embodied Cognition. Singapore: World Scientific.

Van der Stelt, O.; Belger, A.; Lieberman, J. (2004). Macroscopic Fast Neuronal Oscilations and Synchrony in Schizophrenia. PNAS, 101(51), 17567 $-17568$.

Van Gelder, T. (1998). Dinamics and Cognition. En Haugeland, J. (Ed.), Mind design II, Cambridge: MIT press.

Wandell, B.; Brewer, A.; Dougherty, R (2005). Visual Field Map Clusters in Human Cortex. Phylosophical Translations of the Royal Society, 360, 693 707.

Winson, J. (2002) The meaning of dreams. En The Hidden Mind, edición especial de Scientific American, $54-61$.

Wuhle, A.; Fahlbusch, J.; Braun, Ch. (2006). Effects of Motor Activity on the Organization of Primary Somatosensory Cortex. Neuroreport, 17(1), $39-43$.

Fecha de Recepción de artículo: 14 de Septiembre 2007

Fecha de Aceptación de artículo: 29 de abril 2008 